\title{
Inclusive Futures
}

Promoting disability inclusion

"Everything is totally

uncertain right now": people with disabilities' experiences of the COVID-19 pandemic in Bangladesh

A qualitative study undertaken as part of the Inclusion Works programme 


\section{institute of development studies}

Report authors: Jackie Shaw, Fatema Akter, Brigitte Rohwerder, Mary Wickenden and Stephen Thompson

This paper has been funded with UK aid from the UK government. The opinions expressed are those of the authors and do not necessarily reflect the views or policies of the UK government or members of the Inclusion Works consortium.

Suggested citation: Shaw, J., Akter, F., Rohwerder, B., Wickenden, M., \& Thompson, S. (2021). "Everything is totally uncertain right now": people with disabilities' experiences of the COVID-19 pandemic in Bangladesh. Brighton: IDS, DOI: 10.19088/IF.2021.006

\section{Contents}

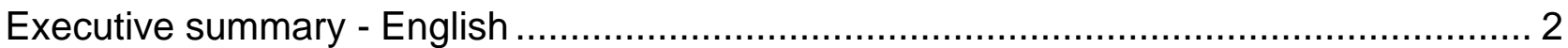

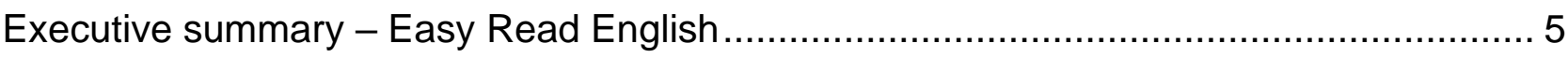

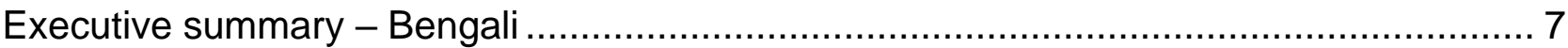

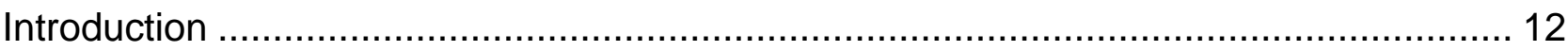

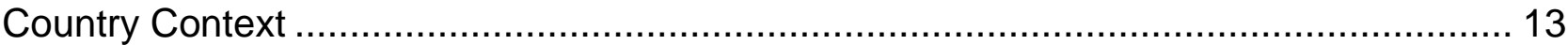

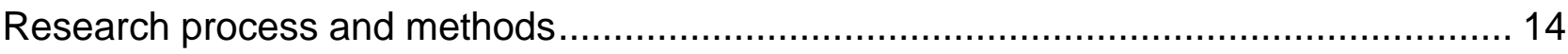

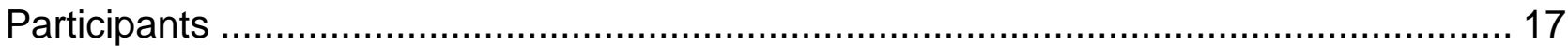

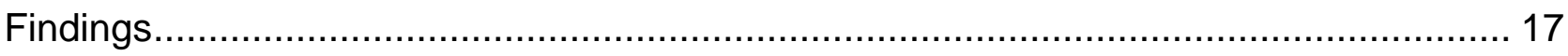

Concrete, material and system related impacts ............................................ 18

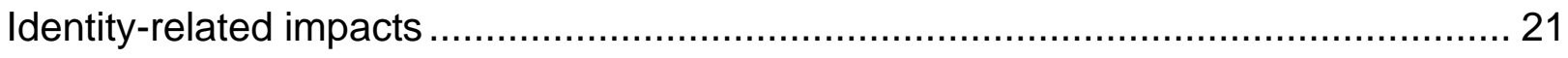

Emotions/feelings (internal or subjective) effects .............................................. 25

Changes over time - concrete circumstances and emotional responses.................. 28

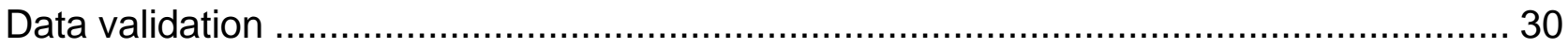

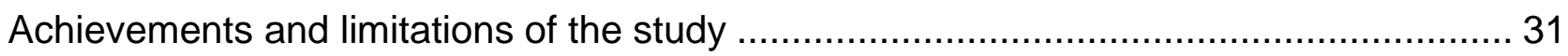

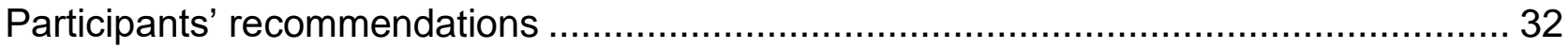

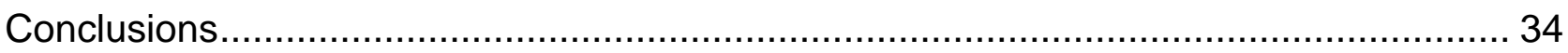

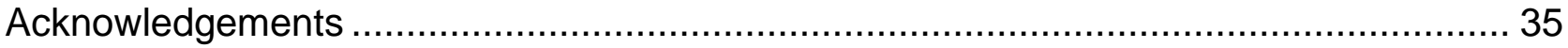

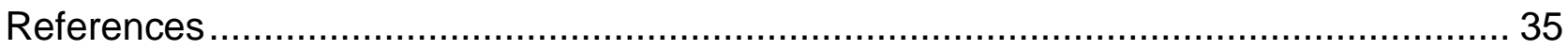

Annex 1 - IW COVID interviews - participants in Bangladesh ................................... 39 


\section{Executive summary - English}

The COVID-19 pandemic is deepening pre-existing inequalities. Emerging research suggests that people with disabilities across the world have experienced various rights violations and been disproportionality affected by the health, economic and social impacts of the COVID-19 pandemic and the responses to it. The aim of this research was to explore how people with disabilities, who often are excluded from research, have experienced the evolving COVID-19 pandemic. In order to better understand how it has affected jobseekers with disabilities, in-depth qualitative research was conducted as part of the Inclusion Works programme in Bangladesh.

\section{Methodology}

A narrative interviewing approach was selected. Telling stories is a natural and universal form of communication and enables participants to make sense of their experiences and focus on what matters to them. Participants were recruited with the help of organisations of people with disabilities (OPDs) and Sightsavers. Out of the 10 participants two had intellectual impairments, two had physical impairments, two had visual impairments, two had multiple impairments and two were classified as 'other' (Dwarfism and Albinism). The interviews were conducted by in-country researchers with support from the IDS team based in the UK. In order to remain safe during the pandemic, the interviews were conducted online or by phone, with reimbursement for data costs and support with technology. Participants' access and communication support needs were also met. Each participant was given the opportunity to take part in two interviews, a few months apart. This enabled participants to feel more comfortable in sharing their experiences and indicated how these changed as the pandemic progressed. Thematic analysis was conducted online by the IDS team and incountry researchers.

\section{Findings}
"I do not know when everything will be normal again... It gives a lot of mental pressure. Sometimes I felt that the inner pain traumatized me inside." (women, visual impairment, IWCOVBDA10)
"We sometime kept ourselves starved due to lack of food. We are right now, totally at the poor level and faced a lot of financial crisis". (woman, physical, IWCOVBDA7)

The participants with disabilities and their families in Bangladesh experienced major negative economic impacts due to lockdowns and other pandemic restrictions and descended into (deeper) poverty as they or those they were dependant on lost their jobs, businesses, or other income. Interviewees reported reduced food consumption and hunger as they no longer had the money to pay for regular meals for themselves and their family, and some feared they would starve. Increased food and transport costs during the pandemic made things more difficult. People also struggled to afford medical costs. This financial crisis caused the interviewees and their families significant stress and mental pressure as they struggled to meet their needs. In order to survive during the worst of the pandemic and 
lockdown, many interviewees relied on financial support from external family members or friends, used up their savings or business capital, or took out loans. However, not everyone had access to these financial coping strategies, and family and friends were often going through the same difficulties and struggled to help each other.

Many people with disabilities in Bangladesh did not receive any assistance from any formal sources, with serious consequences for their survival. Government social protection assistance, which was a mix of existing social protection programmes and COVID-19 specific assistance, was not provided to many people with disabilities in this study during the pandemic. None of the participants with disabilities in this study had received COVID-19 government support, and there were also complaints that usual assistance was insufficient for basic needs, regular or being provided late. Non-Government Organisations (NGOs) and in particular OPDs played a vital role in supporting some people with disabilities cope, but these organisations struggled with lack of funds to do this work, and only two of our cohort received OPD financial aid. Some people with disabilities expressed disappointment with how little organisations for or of people with disabilities did to support them and others during this time.

Access to healthcare was an issue for some people with disabilities, with clinics closed. This made it hard for some people with disabilities to access doctors or needed medicines with serious consequences in some cases. The lack of protective equipment made some doctors reluctant to treat patients with disabilities. The loss of income and disruption of gender roles caused family tensions, arguments and the perception of increased violence at home. Gender-based violence was also observed to have risen in the wider community during the pandemic.

Negative attitudes towards people with disabilities is deeply ingrained within Bangladesh, and overt discrimination was something people with disabilities experienced normally. However, this study suggested that stigma was exacerbated during the pandemic, with increased mocking, taunting and street harassment experienced particularly for those with a 'visible' impairment. Discriminatory attitudes also affected access to physical support and transport.

The abrupt suspension of the 'old normal', the dramatic life changes, and the need to adapt meant that many participants experienced intense shock and fear as their usual life was completely disrupted. The loss of the stability of usual lives resulted in disorientation and uncertainty about what would happen to them and their families, while the restrictions meant that life was lonely. People's usual support structures were affected which made it harder for them to cope. People felt hopeless, helpless and depressed in the worst times of the crisis. Some people found comfort in religion and trusting to God to get them through the situation.

As restrictions eased and interviewees or their families were able to find work, life for some participants improved as they were able to meet some of their family's immediate needs. Others also felt happier and more hopeful as they were less worried about COVID-19. However, many were unable to find work as restrictions lifted, and were still struggling to survive or thrive. Whilst the acute distress changed over time as people adjusted to the situation, the pandemic is not over, and so the uncertainty and difficulties continue.

\section{Conclusion}


The COVID-19 pandemic and government responses to it have had devastating and lifechanging detrimental effects on people with disabilities. Disability inclusive approaches to the pandemic must be developed together with people with disabilities to ensure that the rights and fundamental freedoms of all persons with disabilities can be realised without discrimination of any kind on the basis of disability. 


\section{Executive summary - Easy Read English}

- We asked 10 people with disabilities in Bangladesh to tell us about their lives during the COVID-19 pandemic. They had different types of disabilities such as intellectual, physical, visual and multiple disabilities. We wanted to ask them as they are not often asked about their lives.

- The COVID-19 outbreak has made life worse for many people with disabilities as well as lots of other people.

\section{What we found out}

- To stay safe from COVID-19 people were told to stay at home, which is called a lockdown. This meant people with disabilities and their families lost their jobs and could not pay for enough food and medicine. This made them very sad and worried.

- They managed by getting money or food from their other family members or friends, or by using the money they had saved, moving back to the land or borrowing money. It was hard to help other people because everyone was struggling.

- Some people got help from the government, organisations of people with disabilities, or charities. But no-one we spoke to got extra money from the government during the pandemic, and many people did not get any help at all.

- COVID-19 has changed how everyone lives. This was a shock for many people and made them unsure about what would happen to them and their families. Staying at home all the time made people unhappy. Not having enough money caused 
conflict in families. People felt scared and hopeless - like there was nothing they could do to make things better.

- After a while it was safer to leave home again. This meant some people got their jobs back and they felt happier as they had money to pay for things like food again. Other people were happy because they got used to how things are now and were less scared.

- But some people still did not have work or money to buy food and they were still sad and worried.

- It is important that the government and other organisations help people with disabilities during the COVID-19 outbreak. They should ask people with disabilities about what they need and how this help should be given to them. 


\section{Executive summary - Bengali}

\section{সারসংক্ষেপ}

কোভিড-১৯ এর কারণে আগে থেকে বিদ্যমান বৈষম্য আরও গভীরতর হচ্ছে। ইদানিংকালের গবেষণা থেকে দেখা যায় যে, সারা বিশে^ই প্রতিবন্ধী ব্যক্তিদের অধিকার বিভিন্নভাবে খর্ব হয়েছে এবং কেভিড-১৯ এর প্রভাবে তারা স্বাস্থ্যগত, অর্থনৈতিক ও সামাজিকভাবে ক্ষতিগ্রস্ত হয়েছে।

এই গবেষণার মূল উদ্দেশ্য ছিল গবেষণার আওতা থেকে প্রায়শই বাদ পড়া প্রতিবন্ধীদের অভিজ্ঞতা এই ক্রমবর্ধমান কোভিড-১৯ পরিস্থিতিতে কেমন ছিল তা সম্পর্কে জানা।

চাকুরিপ্রত্যাশী প্রতিবন্ধীরা এই পরিস্থিতিতে কিভাবে প্রভাবিত বা ক্ষতিগ্রস্ত হয়েছে তা ভালো ও স্পষ্টভাবে বোঝার জন্য বাংলাদেশে ইনক্লুশন ওয়ার্কস প্রোগ্রামের অংশ হিসেবে একটি বিশদ কোয়ালিটিটিভ বা গুণগত গবেষণা পরিচালিত হয়।

\section{পদ্ধতি}

গবেষণার পদ্ধতি হিসেবে বিশদ বর্ণনামূলক সাক্ষাৎকার পদ্ধতি বা ন্যারেটিভ ইন্টারভিউইং এপ্রচ গ্রহন করা হয়। গল্প হচ্ছে যোগাযোগ স্থাপনের একটি সহজাত এবং সর্বজনীন মাধ্যম যার সাহায্যে অংশগ্রহনকারী বা গল্পকথক নিজের অভিজ্ঞতাকে অন্যের কাছে সহজবোধ্য করে উপস্থাপন করতে পারেন এবং তার কাছে মূলত কোন বিষয়টি গুরুত্বপূর্ণ তা বোঝাতে পারেন।

অর্গানাইজেশনস অব পিপল উইথ ডিসএবিলিটিজ (ওপিডি) বা প্রতিবন্ধী ব্যক্তিদের দ্বারা গঠিত সংস্থা এবং সাইটসেভার্স-এর সহযোগীতায় গবেষণার জন্য অংশগ্রহণকারীদেরকে নির্বাচন করা হয়।

দশজন অংশগ্রহণকারীর মধ্যে দুজন ছিলেন বুদ্ধি প্রতিবন্ধী, দুজন শারীরিক প্রতিবন্ধী, দুজন দৃষ্টি প্রতিবন্ধী, দুজন অংশগ্রহনকারীর ছিল একাধিক প্রতিবন্ধীতা এবং দুজন অংশগ্রজনকারীকে "অন্যান্য" শ্রেণির অন্তর্ভূক্ত করা হয়েছিল; "অন্যান্য" শ্রেণির অন্তর্ভূক্তদের মধ্যে একজন ছিলেন খর্বাকৃতি এবং অন্যজন শ্বেতিরোগগ্রস্ত ছিলেন।

যুক্তরাজ্যের গবেষণা সংস্থা আইডিএস (IDS) এর সহযোগীতায় দেশীয় গবেষকেরা এই গবেষণার জন্য প্রয়োজনীয় সাক্ষাৎকারগুলো গ্রহন করেছিলেন।

মহামারী চলাকালীন সময়ে সুরক্ষা বজায় রাখার জন্য, সাক্ষাৎকারগুলো অনলাইনে কিংবা ফোনের মাধ্যমে নেওয়া হয়েছিল। সাক্ষাতকার প্রদানের জন্য অংশগ্রহনকারীর প্রয়োজনীয় ব্যয় (ইন্টারনেট ডেটা প্যাকের খরচ) এবং প্রযুক্তিগত সহায়তা প্রকল্প থেকে দেওয়া হয়েছিল। এছাড়াও অংশগ্রহনকারীর অভিগম্যতা নিশ্চিতকরণ এবং যোগাযোগ স্থাপনের জন্য প্রয়োজনীয় সহযোগীতাও প্রকল্প থেকে দেওয়া হয়েছিল।

প্রত্যেক অংশগ্রহণকারী কয়েক মাসের অন্তর দুইটি আলাদা সাক্ষাৎকারে অংশগ্রহণ করার সুযোগ পেয়েছিলেন। এর ফলে তারা আরও স্বাচ্ছন্দ্যে তাদের অভিজ্ঞতার কথা জানাতে পেরেছেন এবং ক্রমবর্ধমান মহামারীর 
প্রেক্ষাপটে তাদের অভিজ্ঞতাগুলো কিভাবে পরিবর্তিত হয়েছে তা তুলে ধরতে পেরেছেন। আইডিএস এবং দেশীয় গবেষকদের সমন্বয়ে অনলাইনে একটি থিম্যাটিক বিশ্লেষণ কার্যক্রম পরিচালিত হয়েছিল।

\section{ফলाফল}

"আমি জানি না সবকিছু আবার কবে স্বাভাবিক অবস্থায় ফিরে যাবে... এর ফলে আমি খুব মানসিক চাপে আছি। কখনও কখনও আমার মনে হয় এই চাপা কষ্ট ভিতরে ভিতরে আমাকে আতঙ্কিত করে রাখছে"। (নারী অংশগ্রহনকারী, দৃষ্টি প্রতিবন্ধী, IWCOVBDA10)

"খাবারের অভাবে অনেক সময়ই আমাদের না খেয়ে থাকতে হয়েছে। এখন আমরা পুরোপুরিভাবেই গরীব এবং আমাদের নানারকম অর্থ সংকটের মধ্য দিয়ে যেতে হচেছ " (নারী অংশগ্রহনকারী, শারীরিক প্রতিবন্ধী, IWCOVBDA7)

এই গবেষণায় অংশ নেওয়া বাংলাদেশী প্রতিবন্ধী এবং তাদের পরিবারের সদস্যরা লকডাউন এবং এই মহামারীর কারণে প্রদত্ত অন্যান্য নিষেধাজ্ঞার কারণে আর্থিকভাবে ভীষণ ক্ষতিগ্রস্ত হয়েছেন; যা তাদের দরিদ্রতা আরও বাড়িয়ে দিয়েছে। কারণ এই লকডাউন এবং অন্যান্য নিষেধাজ্ঞার ফলে অংশগ্রহনকারী অথবা তাদের পরিবারের সদস্য যাদের উপর তারা নির্ভরশীল, হয় চাকরি হারিয়েছেন নয়তো ব্যাবসা ও আয়ের অন্যান্য পথ হারিয়েছেন।

গবেষণায় অংশগ্রহনকারীরা সাক্ষাৎকারের সময় "খাদ্যগ্রহন সীমিতকরণ" এবং "ক্ষুধা" এর কথা উল্লেখ করেন; তারা জানান, তাদের নিজেদের এবং পরিবারের জন্য প্রয়োজনীয় খাবার কেনার টাকা তাদের কাছে ছিলনা বলে এমন পরিস্থিতি ঘটে এবং এর ফলে তাদের কেউ কেউ অনাহারে থাকার ভয়েও ভীত ছিলেন।

মহামারী চলাকালীন সময়ে খাবার এবং পরিবহনের খরচ বেড়ে যাওয়ায় পরিস্থিতি আরও কঠিন হয়ে পড়ে। এই পরিস্থিতিতে চিকিৎসার খরচ চালাতেও মানুষকে হিমশিম খেতে হয়েছে। সাক্ষাৎকারে অংশগ্রহনকারীরা আরও জানান যে, সেই সময় তারা এবং তাদের পরিবারের সদস্যদের পক্ষে তাদের নিত্যদিনের চাহিদা মেটানোই মুশকিল হয়ে পড়েছিল; আর সেই কারণে তাদেরকে দুশ্চিন্তা ও মানসিক চাপের মধ্য দিয়ে যেতে হয়েছে।

গবেষণা থেকে প্রাপ্ত তথ্য মতে, লকডাউন পরিস্থিতিতে টিকে থাকার জন্য গবেষণায় অংশগ্রহনকারীদের অনেকেই তাদের পরিবারের বাইরের কেউ বা বন্ধু-বান্ধবের সাহায্যের উপর নির্ভর করেছেন, কেউ তাদের জমান অর্থ বা ব্যাবসার পুঁজি ভাঙ্গিয়েছেন অথবা কেউ কেউ ঋণ নিয়েছেন। তবে, এই আর্থিক সংকটের মোকাবেলা করার জন্য এই ধরনের উপায় সবার ছিল না; এবং প্রায় ক্ষেত্রেই তাদের পরিবারের অন্যান্য সদস্য এবং বন্ধুরাও একই রকম কঠিন পরিস্থিতির মধ্যে দিয়ে যাচ্ছিলেন তাই এই অবস্থায় একে অপরকে সাহায্য করাও অনেক ক্ষেত্রে মুশকিল ছিল।

বাংলাদেশের বহু প্রতিবন্ধী এই সঙ্কটেও কোনও প্রাতিষ্ঠানিক উৎস থেকে কোনও ধরনের সহযোগীতা পাননি; যার ফলে এই সঙ্কটময় পিরিস্থিতিতে টিকে থাকা তাদের জন্য খুব কঠিন হয়ে পড়ে। গবেষণায় অংশগ্রহনকারীদের অনেকেই মহামারী পরিস্থিতি চলাকালীন সময় সরকার কর্তৃক সামাজিক নিরাপত্তা কর্মসূচী ও কোভিড- ১৯ এর জন্য গ্রিহিত জরুরি সহায়তা কর্মসূচীর আওতায় প্রদত্ত কোন সহায়তাই পাননি। অংশগ্রহনকারীরা জানান যে, তাদের মধ্যে কেউই কোভিড- ১৯ এর জন্য সরকারী জরুরি সহায়তা কর্মসূচীর আওতায় প্রদত্ত কোন সহায়তাই পাননি; তারা আরও অভিযোগ করেন যে, প্রতিবন্ধীদের জন্য প্রদত্ত নিয়মিত সরকারী সহযোগীতাও তাদের মৌলিক চাহিদা মেটানোর জন্য যথেষ্ট নয় এবং নিয়মিভাবে বা সময় মত তারা তা পানও না। 
এই পরিস্থিতিতে কিছু প্রতিবন্ধী ব্যক্তিকে সহয়তার জন্য এনজিও বিশেষত ওপিডি একটি গুরুত্বপূর্ণ ভূমিকা পালন করেছে। কিন্তু সংস্থাগুলোর এই কাজের জন্য যথেষ্ট ফান্ড ছিলনা; ফলে, মাত্র দুজন সদস্য ওপিডি ফান্ডের আওতায় এই সহযোগীতা পেয়েছিল। এধরনের অপ্রতুল সহায়তা প্রদানের জন্য গবেষণায় অংশগ্রহনকারীরা তাদের হতাশা প্রকাশ করেন।

লকডাউনে ক্লিনিক বন্ধ থাকার কারণে কিছু প্রতিবন্ধী ব্যক্তির জন্য স্বাস্থ্যসেবা পাওয়া কঠিন হয়ে পড়েছিল; এই পরিস্থিতিতে তারা প্রয়োজনে ডাক্তার দেখান বা ওযুধ সংগ্রহ করতে পারছিলেননা যার ফলে কেউ কেউ মারাত্মক সমস্যার সম্মুখীন হয়েছিলেন। কোভিড- ১৯ থেকে রক্ষা পাবার জন্য প্রয়োজনীয় সুরক্ষা সরঞ্জামের অভাবে কোন কোন চিকিৎসক প্রতিবন্ধী ব্যক্তিদের চিকিৎসায় অনাগ্রহ প্রকাশ করেন। লকডাউনে আয় কমে যাওয়া বা পরিবারে জেন্ডার ভূমিকায় ব্যাঘাত ঘটার কারণে পরিবারগুলোতে উত্তেজনা বা ঝগড়া-ঝাটির ঘটনা ঘটতে শুরু করে; যার ফলে বাসা-বাড়িতে সহিংসতার ঘটনাও বেড়েছে বলে ধারণা করা হচ্ছে। এই পরিস্থিতিতে সমাজে জেন্ডার ভিত্তিক সহিংসতা বৃদ্ধির ঘটনাও একটি লক্ষণীয় বিষয়।

বাংলাদেশের অনেকেই প্রতিবন্ধীদের প্রতি ভীষণ নেতিবাচক ধারণা পোষণ করে এবং প্রতিবন্ধীরা প্রায়ই বিভিন্ন ধরনের বৈষম্যের শিকার হন। তবে এই গবেষণায় দেখা যায় যে, এই মহামারী চলাকালীন সময়ে প্রতিবন্ধী ব্যক্তিদের প্রতি বৈষম্য বা অপমানকর আচরণ আরও বেড়ে গেছে। প্রতিবন্ধী ব্যক্তিদেরকে আজে-বাজে কথা বলা, উত্যাক্ত করা, রাস্তা-ঘাটে হয়রানীর ঘটনা বেড়ে গেছে; বিশেষত দৃষ্টি প্রতিবন্ধীরা এ ধরনের ঘটনার সম্মুখীন বেশি হচ্ছেন। বৈষম্যমূলক দৃষ্টিভঙ্গি প্রতিবন্ধীদের জন্য বিভিন্ন ধরনের অবকাঠামো বা যানবাহনে অভিগম্যতা নিশ্ছিত করার ক্ষেত্রে একটি বড় বাধা।

স্বাভাবিক জীবনের আকষ্মিক পরিবর্তন ও তার সাথে খাপ খাওয়াতে গিয়ে গবেষণায় অংশগ্রহনকারীদের অনেকেই প্রচণ্ড চাপের সম্মুখীন হয়েছেন। স্বাভাবিক জীবন যাপনে অস্থিতিশীলতা সৃষ্টি হবার কারণে প্রতিবন্ধী ব্যক্তি এবং তাদের পরিবারের সদস্যদের ভিতর এক ধরনের অনিশ্চয়তা দেখা দিয়েছে: এর একটা বড় কারণ হল মহামারীর কারণে প্রদত্ত বিভিন্ন বিধি-নিষেধের ফলে মানুষ একা হয়ে পড়েছে। তাদের জীবন ধারণের জন্য প্রয়োজনীয় স্বাভাবিক সব উপায়ই এই সংকটময় পরিস্থিতিতে ক্ষতিগ্রস্ত হয়েছে যার ফলে পরিস্থিতির সাথে খাপ খাওয়ানো তাদের জন্য আরও কঠিন হয়ে পড়ে। চরম সংকটময় সময়গুলোতে মানুষ ভীষণভাবে নিরাশা, হতাশা ও বিষন্নতায় ভুগছিল। এই পরিস্থিতিতে ধর্মের প্রতি বিশ্বস তাদের শান্তি দিয়েছিল; তারা বিশ্বাস রেখেছিলেন যে স্রষ্টা অবশ্যই এই খারাপ সময় কাটিয়ে উঠতে সাহায্য করবেন।

বিধি-নিষেধ কিছুটা শিথিল হওয়ায় গবেষণায় অংশগ্রহনকারী এবং তাদের পরিবারের সদস্যরা ধীরে ধীরে চাকরি বা কাজ পাচ্ছিলেন; এর ফলে তারা জীবন ধারণের জন্য প্রয়োজনীয় চাহিদা মেটাতে পারছিলেন যার ফলে কয়েকজন অংশগ্রহনকারীর অবস্থার কিছুটা উন্নতি হয়েছিল। সেই সময় কোভিড- ১৯ নিয়ে দুশ্চিন্তা কমে যাওয়ায় অন্যান্যরাও আগের চেয়ে ভাল বোধ করছিলেন এবং তাদের মনে আশার সঞ্চার হয়েছিল। তবে নিষেধাজ্ঞা তুলে নেবার পরও কারও কারও জন্য কাজ পাওয়া বা টিকে থাকা অত্যন্ত কঠিন ছিল। যদিও সময়ের সাথে সাথে সংকটের তীব্রতা কমেছে এবং মানুষও ধীরে ধীরে অভ্যস্ত হয়ে পড়েছে তবুও এটা সত্যি যে, মহামারী এখনও শেষ হয়ে যায়নি এবং মানুষের জীবনের অনিশ্চয়তা এবং সসমস্যা এখনও বিদ্যমান।

\section{উপসংহার}

কোভিড- ১৯ এবং এর জন্য গ্রিহিত বিভিন্ন সরকারী পদক্ষেপ প্রতিবন্ধী জনগোষ্ঠির জীবনে ভয়াবহ ক্ষতিকর প্রভাব ফেলেছে। এই কারণেই এই মহামারী পরিস্থিতিতে বৈষম্যহীনভাবে প্রতিবন্ধীদের অধিকার ও মৌলিক স্বাধীনতা নিশ্চিত করার লক্ষ্যে প্রতিবন্ধী জনগোষ্ঠিকে সাথে নিয়েই একটি "ডিসএবিলিটি ইনকৃলুসিভ এপ্রচ" বা প্রতিবন্ধীদের জন্য সমন্বিত পদক্ষেপ গ্রহন করতে হবে। 


\section{নির্বাহী সারসংক্ষেপ - সহজ পঠনীয় - বাংলা}

- বাংলাদেশে বসবাসরত ১০ জন প্রতিবন্ধী ব্যক্তির কাছ থেকে কোভিড- ১৯ চলাকালীন সময়ে তাদের জীবন যাপন বা তাদের অবস্থা কেমন ছিল তা জানতে চাওয়া হয়েছিল। অংশগ্রহনকারীদের মধ্যে কেউ ছিলেন বুদ্ধি প্রতিবন্ধী, কেউ শারীরিক প্রতিবন্ধী, কেউ দৃষ্টি প্রতিবন্ধী এবং কারও কারও ছিল একাধিক প্রতিবন্ধীতা। তাদেরেকে তাদের জীবন সম্পর্কে জিজ্ঞাসা করার কারণ হল অধিকাংশ ক্ষেত্রেই তাদের জীবন সম্পর্কে কেউ জানতে চায়না।

- প্রতিবন্ধী জনগোষ্ঠি সহ সবার জীবনেই কোভিড-১৯ মারাত্মক ক্ষতিকর প্রভাব ফেলেছে।

\section{আমরা কী জানতে পেরেছি}

- কোভিড- ১৯ থেকে সুরক্ষিত থাকার জন্য লোকজনকে বাসায় থাকার নির্দেশ দেওয়া হয় যা লকডাউন নামে পরিচিত। এর ফলে প্রতিবন্ধী ব্যক্তি ও তাদের পরিবারের সদস্যরা তাদের চাকরি হারায়; যার ফলে সেই সময় প্রয়োজনীয় খাবার বা ওষুধ কেনার মত যথেষ্ট টাকা তাদের হাতে ছিলনা। এই অবস্থা তাদের মাঝে এক ধরনের উদ্বেগ সৃষ্টি করে।

• এই পরিস্থিতি মোকাবেলা করার জন্য তাদেরকে পরিবারের অন্যান্য সদস্য বা বন্ধুবান্ধবের কাছ থেকে টাকা, খাবার ইত্যাদি সাহায্য হিসেবে নিতে হয়েছিল। কেউ কেউ নিজের সঞ্চয় ভেঙ্গেছিল, কেউ গ্রামে ফিরে গিয়েছিল অথবা কেউ টাকা ধার করেছিল। কিন্তু এই পরিস্থিতিতে কাউকে সাহায্য করা খুব কঠিন ছিল। কারণ প্রত্যেকেই সমস্যার মধ্য দিয়ে যাচ্ছিলেন।

• স সরকার, প্রতিবন্ধীদের জন্য গঠিত সংস্থা (ওপিডি) বা বিভিন্ন দাতব্য সংস্থা থেকে কেউ কেউ কিছু সহযোগীতা পেয়েছিলেন। কিন্তু আমরা যাদের সাথে কথা বলেছি তাদের কেউই মহামারী চলাকালীন সময়ের এই বিশেষ সংকট পার করার জন্য সরকারের কাছ থেকে কোনও অতিরিক্ত আর্থিক সহযোগীতা পাননি। এবং অধিকাংশই কোনও ধরনের সহযোগীতাই পাননি। 
- কোভিড- ১৯ প্রত্যেকের জীবন পাল্টে দিয়েছে। অনেকের জন্যই এটি ছিল একটি বড় ধরনের ধাক্কা; তাদের নিজেদের এবং পরিবারের কী হবে তা নিয়ে তারা অনিশ্চয়তায় ভুগছিলেন। সারাক্ষণ বাসায় বসে থাকায় মানুষ হাঁপিয়ে উঠেছিল। হাতে টাকা-পয়সা না থাকায় পরিবারগুলোতে ঝগড়া-ঝাটি লেগে যাচ্ছিল। মানুষ ভীত-সন্ত্রস্ত ও হতাশ হয়ে পড়ছিল। তাদের মনে হচ্ছিল, এই পরিস্থিতি ঠিক করার কোনও উপায়ই যেন তাদের হাতে নেই।

- কিছুদিন পর কোভিড পরিস্থিতি কিছুটা নিয়ন্ত্রনে এলে মানুষ আবার বাড়ির বাইরে যেতে শুরু করে। এর ফলে কেউ কেউ তাদের চাকরি ফিরে পায় যার ফলে তারা খাবার সহ অন্যান্য নিত্য প্রয়োজনীয় জিনিসের ব্যয়ভার গ্রহন করতে পারে। ফলে তাদের ভিতর কিছুটা স্বস্তি ফিরে আসে। পরিস্থিতির সাথে অভ্যস্ত হয়ে যাওয়ায় মানুষের মাঝে ভয়-ভীতি কমে গিয়েছিল; যার ফলে অন্যরাও স্বস্তি বোধ করছিল।

- িন্তু কিছু লোকের কাজ বা চাকরি না থাকায় খাবার বা অন্যান্য প্রয়োজনীয় সমগ্রী কেনার মত টাকা তাদের হাতে ছিলনা; ফলে সেই সময়ও তারা উদ্বিগ্ন ছিল।

- কোভিড- ১৯ চলাকালীন সময় সরকার ও অন্যান্য সংস্থার পক্ষ থেকে প্রতিবন্ধী ব্যক্তিদের প্রতি সহযোগীতার হাত বাড়িয়ে দেওয়া অত্যন্ত প্রয়োজন। প্রতিবন্ধী ব্যক্তিদের কী ধরনের এবং কিভাবে সহযোগীতা করা প্রয়োজন তা প্রতিবন্ধী ব্যক্তিদের কাছ থেকেই জানতে হবে। 


\section{Introduction}

The COVID-19 pandemic is deepening pre-existing inequalities, including those faced by people with disabilities (UN, 2020). At the beginning of the COVID-19 pandemic serious concerns were raised about the possible impacts of the pandemic for people with disabilities and their families, and whether the national and local support for populations during the pandemic would be sufficiently disability inclusive (IDA, 2020; UN, 2020). Guidelines were produced by key organisations suggesting ways to avoid the predicted exacerbation of the disadvantaged position of people with disabilities in many contexts (IDA, 2020; IDA and IDDC, 2020; ILO, 2020a and b; Inclusion International, 2020; OHCHR, 2020; WHOa, 2020; WHOb 2020).

Initially information about the situation for people with disabilities was scarce, but as the pandemic has progressed emerging evidence indicates that they have experienced various rights violations and been disproportionality affected by the health, economic and social impacts of COVID-19 (COVID-19 DRM, 2020). People with disabilities are especially at risk of catching and dying from COVID-19, due to a combination of lack of accessible information about COVID-19 prevention, increased barriers to health care, reduced support or care, or, for some, increased clinical vulnerability (Webster, 2020; HI, 2020a; Goyal et al., 2020; COVID-19 DRM, 2020; i2i, 2020; HI, 2020b). Barriers to education, already present for children with disabilities, have increased with school closures and the move to online learning has generally not considered their access needs (Goyal et al., 2020; COVID-19 DRM, 2020; Nagari, 2020). Various surveys have found that the pandemic has negatively affected the incomes of people with disabilities and their households as they have lost their jobs, resulting in increased food insecurity (HI, 2020b; HI, 2020a; Goyal et al., 2020; i2i, 2020; Ahmed et al, 2020; Christensen, 2020a; Christensen, 2020b). Pre-existing disability related stigmatisation, discrimination and violence are reported to have increased during the outbreak (HI, 2020a; UN, 2020; Goyal et al., 2020; COVID-19 DRM, 2020; Christensen, 2020a, WEI 2020; Christensen, 2020b), and people with disabilities' mental health has been severely affected by the enforced isolation of lockdowns and other restrictions (COVID-19 DRM, 2020; Sale et al 2020; Goyal et al., 2020). These emerging findings suggest the global pandemic has led to the predicted negative consequences for people with disabilities. However, more evidence is needed to fully understand what has happened to people from different impairment groups and living in diverse situations across the world during the pandemic. In particular, there is limited in-depth understanding about how people with disabilities in low-and-middle income countries have experienced the evolving COVID-19 situation from their perspective.

The Inclusion Works (IW) programme, funded by the UK's Foreign, Commonwealth, and Development Office (FCDO), involves a consortium of 10 organisations working in four countries over four years (2018-2022). It aims to create and test innovative approaches to improve the long-term economic empowerment and inclusion of people with disabilities in formal employment. The programme has responded to the ongoing COVID-19 pandemic in a variety of ways, including this research, which has been conducted through collaboration with international and local partners, including Organisations of Persons with Disabilities 
$\left(\mathrm{OPDs}^{1}\right)$, and in line with UNCRPD principles. The overall research aim was to better understand how people with disabilities have experienced the evolving COVID-19 pandemic in the four countries involved in the Inclusion Works programme - Bangladesh, Kenya, Nigeria, and Uganda. This report highlights the findings of this in-depth qualitative research with jobseekers with disabilities involved with the Inclusion Works programme in Bangladesh. The study explored their lived experiences over two different phases of the COVID-19 pandemic using a narrative interviewing approach, which involved them directly in communicating their experiences and focused on their priorities and perceptions, as well as gathering their own recommendations on pandemic responses.

\section{Country Context}

A new coronavirus disease (COVID-19) was first identified in December 2019, with the WHO declaring the outbreak a pandemic on $11^{\text {th }}$ March 2020. The first three known cases in Bangladesh were reported three days prior on $8^{\text {th }}$ March 2020 by the country's Institute of Epidemiology Disease Control and Research (IEDCR) (Paul, 2020). To protect the population, the Government declared a national lockdown starting on $23^{\text {rd }}$ March. Initially it was due to finish after 10 days but was soon extended to $30^{\text {th }}$ May. Infections remained low until the end of March but saw a steep rise in April (Nabi and Shovon, 2020). In the week ending on $11^{\text {th }}$ April, new cases grew by 1,155 percent, the highest in Asia, ahead of Indonesia with 186 percent (Devulapalli \& Dantewadia, 2020). As of 25 $5^{\text {th }}$ March 2021, in Bangladesh there have been 580,808 confirmed cases of COVID-19 with 8,763 deaths reported to WHO (WHO 2021). Bangladesh is the second most affected country in South Asia, after India.

In the early stages of the crisis, the government of Bangladesh was slow to respond to demands for testing. By the end of March (a week into the national lockdown), only 1,185 people had been tested for COVID-19 in Bangladesh. By the same date, neighbouring India had carried out 27,688 tests and Pakistan 14,336 (Alif, 2020). Newspaper reports and social media continued to report deaths of patients with COVID-19 symptoms. Some of the deceased were treated at COVID-19 isolation centres at hospitals in the districts, though no tests were conducted to confirm infection (Maswood, 2020). In the early days, testing remained centralised at the IEDCR, which is based in the capital Dhaka, although patients with symptoms were reported all around the country. The IEDCR created a series of hotline numbers, email address and social media pages for people to contact them if they had COVID-19 symptoms and to provide information.

Bangladesh has faced significant challenges in combating COVID-19, as it is a densely populated country, and also houses a million Rohingya refugees in sprawling refugee camps conducive to the spread of disease. It also has significant migrant populations living in

${ }^{1}$ Organisations of Persons with Disabilities (OPDs) are also sometimes referred to as Disabled People's Organisations (DPOs) 
various countries, including China and Italy, which were badly affected by COVID-19 in early 2020. Religion is an important part of Bangladeshi culture, and mass religious events and communal prayers may have helped to spread the virus (Nooruddin \& Shahid, 2020).

The economy in Bangladesh has been badly affected by the pandemic. Exports from the ready-made garment industry are a major part of Bangladesh's economy, and demand for this industry has been particularly badly hit. COVID-19 has caused unemployment, job losses, reduced incoming remittances, food insecurity and poverty in Bangladesh. The socioeconomic and development impacts have been severe, and the health sector is under considerable strain. The pandemic will have a detrimental impact on working towards the targets of Sustainable Development Goals. In addition, quality education will be hampered in the country, although institutions are now open and some are exams going ahead. Some are not and students have been protesting about this. The government of Bangladesh has mobilised a stimulus package to support the affected industries and communities. However, recent research found that government support (both COVID-19 specific and from prior programmes) was not provided to many people with disabilities during the pandemic (Rohwerder et al, 2021). Non-Government Organisations (NGOs) and in particular OPDs have played an important role in supporting people with disabilities but are under resourced. It is not yet clear how services such as education and health will be strengthened and supported.

As of January 2021, there were no lockdown measures in place. There is concern that natural disasters which are common in Bangladesh, including cyclones, tidal floods, flash floods, and landslides are likely in the coming months and could add to the challenges posed by COVID-19 and further aggravate the humanitarian needs of the most vulnerable groups in the country.

\section{Research process and methods}

This study focussed on involving participants who were job seekers with disabilities in the Inclusion Works programme in Bangladesh. We aimed to involve those from the most marginalised impairment groups, who are often excluded from research. In Bangladesh this included people with intellectual and multiple impairments, dwarfism and albinism.

With the UNCRPD as a guiding framework, a research design was developed using qualitative methodologies, as these were deemed to be most appropriate for generating subjective knowledge from within a context (Tracey, 2013; Braun and Clarke, 2012; Hammett et al, 2015). A narrative interviewing approach was selected for several reasons:

- Telling stories is a natural and universal form of communication - this method is accessible to everyone regardless of education level, language competence or communication mode (Jovchelovitch and Bauer, 2000).

- By recollecting and sequencing experiences as stories, participants make sense of their experiences. 
- As a teller-focused method, evidence about what matters to participants is generated, rather than using pre-determined questions to investigate topics that may be of less concern to them (Hydén, 2014).

- This approach can generate insights on situational, emotional and relational aspects, that may not emerge from researcher-directed interviews.

Each participant was given the opportunity to take part in two rounds of interviews, separated by a few months. The purpose of undertaking two rounds of interviews was first to gain deeper insight both as trusting relations and thus openness developed between participants and researchers, and because the narratives were anticipated to flow more easily as the interviewees' expressive confidence increased. Second, this provided an additional processual lens, to enable some insight on how disabled participants interpreted and responded to COVID-19 as the pandemic progressed over time.

An experienced participatory researcher from Bangladesh led the data collection process. The Institute of Development Studies (IDS) team developed the research processes and materials, provided training and supervisory accompaniment for the local researcher, and led the analysis process. Extra researcher training on dealing with sensitive issues and the communication needs for different impairment groups was provided by the wider team and partners.

Participants were recruited from the pool of jobseekers with disabilities involved in some way with the Inclusion Works programme, including those from often excluded populations, such as people with intellectual disabilities. Support from the Sightsavers and ADD International Bangladesh offices, Access Bangladesh Foundation and other Organisations of People with Disabilities (OPDs), was crucial to reaching these participants. The local researchers worked with them to purposively reach beyond those usually asked to participate, and to ensure inclusion of a range of participants, a gender-balance, and a range of ages.

To ensure that both the participants and researcher remained safe during the research process, the narrative interviews were undertaken online or by phone, which is an accepted approach (Holt, 2010). The researcher was encouraged to conduct interviews on online communication platforms such as TEAMs or Zoom and use the video function when possible. This was both to aid relationship building and effective communication, which was particularly important for those such as the participants with intellectual disabilities, who may rely more on body language, gestures, or facial expressions. Data costs were reimbursed. The choice of platform was ultimately informed by each individual participant's preferences, capacities and access to technology.

Ethics approval for this work was obtained from the IDS ethics committee. To mitigate heightened ethical risks compared with face-to-face interviewing (such as the additional difficulties of reading dynamics or responding supportively if strong emotions arose), the research process involved three calls with each participant during the first interview round:

On the first call the research purpose and process were explained, including consent and anonymisation, the right to withdraw, and the analysis and reporting plans. The participant's 
access and communication support needs were also discussed prior to the interview, which enabled necessary accommodations to be made.

On the second call participants were asked for verbal consent to be involved. This gave them time and space after the first call to decide whether to participate, reducing any perceived pressure to participate. The interview was conducted after verbal consent was gained and the participant confirmed that they felt secure, and their access needs were being met. After an initial introduction, a narrative interview proceeds by asking one very general and open question (Jovchelovitch and Bauer, 2000):

\section{Participants were asked to share how the COVID situation had developed for them and impacted on their lives.}

The interviewer reassured them that they could share experiences in any way they liked but encouraged the story-form by suggesting they start at the beginning, when they first heard about the pandemic, and then relate what happened afterwards up to the present. Prompts and follow-up questions were used to ask for specific examples, or to expand on the key aspects that interviewees chose to talk about. Finally, there was a third call to ensure that the participant was feeling alright and still happy to be involved.

The second round of interviews followed a similar process. It was anticipated that the second round of interviews would involve greater openness and narrative flow due to developing relations between the researcher and participant.

A face-to-face participatory thematic analysis process was planned, involving the researcher and some participants. However, this plan had to be adapted due to ongoing COVID-19 restrictions. To minimise risk and comply with regulations, a series of collective analysis sessions were undertaken online, involving the IDS team and the in-country researchers from the four countries. Prior to undertaking the analysis, the researchers were provided with thematic analysis training.

The analysis sessions included an initial reading of the first interviews, and the sharing of interview narratives. From the data, themes were generated and compared across countries. Following discussion and debate, the themes were then clustered to produce a first-level analysis. Overarching themes, such as gender, impairment specific aspects, emotions and economic/financial impacts were established. The IDS team-members each analysed the remaining interviews using one of these lenses. After the second round of interviews had been conducted, a second analysis session was held, where additional themes were generated based on the current situation in each country. New insight was compared with the detailed first round analysis. This validated previous themes and indicated data saturation, but there were also changes in impacts and feelings, as well as some additional overarching themes. Collectively generating overarching meanings and interpretations, ensured that this synthesis was grounded in local perspectives. 


\section{Participants}

A table detailing the characteristics of the Bangladeshi participants can be found in Annex 1.

In total 10 participants took part, with a 50:50 gender balance between females and males. Participants were recruited with the help of organisations of people with disabilities (OPDs) and the IW consortium partners ADD International and Sightsavers. Two out of the 10 participants had intellectual impairments, two had physical impairments, two had visual impairments, two had multiple impairments and two were classified as 'other' (Dwarfism and Albinism). The participants were mostly young (aged between 18 and 26), with one 32 yearold. They were a well-educated cohort with eight having attended tertiary education (college or university), with the others special education. One participant was an assistant trainer, but most were not in employment. Six were currently students, and three had no occupation or employment. Two participants were married, and the rest were single. Half lived in an urban and half a rural area. Two lived alone, but eight lived with at least one parent, and six with other siblings as well. The largest family was six (two participants). All but one participant was interviewed twice.

\section{Findings}

In this paper we present key themes which emerged from the narrative interviews in Bangladesh. Two different types of interlinking themes emerged: overarching 'subjective' experiences themes and more 'concrete' material impacts.

'Concrete' (material) themes illustrating the tangible impacts were clearly identified. These fall into two main types, with links and overlaps between them. There are the economic/financial impacts and people's experience of the structures, systems and civil society responses to the pandemic. Then there are individual (and intersecting) identity related experiences linked to people's gender and/or impairment type and disability status.

The 'subjective' experience themes broadly reflect the participants' feelings and experiences during the initial COVID-19 crisis and its continuation over a couple of months. These emotions arose in response to the loss of the stability of known life, the 'old normal', the pandemic risks and the lockdown restrictions, and the uncertain journey towards a 'new normal'. We interpreted these emotions as 'floating above' and 'weaving through' participants' experiences expressed as more 'concrete' or material themes. Overall, strong themes of de-stabilisation, disorientation and uncertainty were experienced due to the extraordinary situation, with subthemes of shock, confusion, fear, loss, stress, conflict, anxiety, despair and depression in various forms.

We describe these themes and bring to the fore the voices of the participants in the following section. Quotes are identified as from individuals by gender and impairment type, and interview ID number ( $A$ or $B$ before the number indicate whether the quote comes from the first $(A)$ or the second $(B)$ interview). 


\section{Concrete, material and system related impacts}

\section{Economic impacts}

Similarly to people across the world, our interviewees with disabilities and their families in Bangladesh experienced major negative economic impacts due to lockdown and other pandemic restrictions, and descended into (deeper) poverty as they or those they were dependant on lost their jobs, businesses, or other income. Some people with disabilities had lost their jobs as a result of staying home due to lockdown or fear of catching COVID-19 at work. Most of the people with disabilities in this cohort belong to lower and lower-middleincome families, and the participants were part of family/household units that generally relied on more than one source of income. Generally, most of the income earners in families had lost their job.

\footnotetext{
"My financial condition was bad before the COVID-19 but after the COVID-19 the condition is now worse." (woman, visual impairment, IWCOVBDA10)

"We are totally dependent on my father's income. My father received his salary once in a two month and it is not sufficient for us to run the family." (man, visual impairment, IWCOVBGB6)
"Our two family income sources have totally stopped... Due to COVID-19, I did not get any students to do tuition - I am totally empty handed right now. Furthermore, my husband's income also decreased." (woman, physical impairment, IWCOVBGA7)

Families where at least one person was still working were in a slightly better position as they still had some income on which to survive, though cuts were still necessary.
"With the help of mine and my brother's income, family costs have been
borne. But for both of us the income amount is much less than the
requirement... monthly food items have had to be cut down." (man,
intellectual impairment, IWCOVBGA1)

Especially affected by the negative economic impacts were those with existing financial pressures prior to the outbreak. These included those who lacked a previous job, which includes many people with disabilities due to existing barriers to employment, those needing to pay for ongoing medicine, those with family dependants, and single parents. One student participant, whose parents were retired and brother had lost their job, noted that:
"My family ... faced lot of challenges and difficulties due to the financial instability ...the economic condition of my family totally broke down." (man, multiple impairments, IWCOVBGA2)

Many interviewees reported hunger and reduced food consumption as they no longer had the money to pay for regular food. Some feared they and their families would starve. 
"Due to the COVID-19, my father's business has totally stopped and he is doing day labourer work. Some day he got some work but other day he did not get any work. The days he has some work...we have some food in our house. But when he did not ...that day we had to keep ourselves starved." (woman, visual impairment, IWCOVBGA10)

"We are passing our day by eating two times a day." (woman, physical impairment, IWCOVBGA3)

Increased food and transport costs due to the pandemic also made buying food more difficult, in addition to the other bills.

"The price of daily commodities has risen very high which is not within our
affordability limit. This situation makes our live more miserable." (man,
physical impairment, IWCOVBGB4)

"They were not able to pay their house rent, other utility bills. My financial condition is bad before the COVID-19 but after the COVID-19 the condition is now worsened." (woman, visual, IWCOVBDA10)

Medical costs for those with medical needs in the family, their own or other family members, were also major concerns. It was also noted that people with disabilities struggled to buy hygiene products used for COVID-19 prevention due to their financial situations.

"I and my family member were not able to take any medical treatment as the expenses are very high. We have to buy medicine from pharmacies without any doctor's consultation which is very dangerous." (man, physical impairment, IWCOVBGB4)

"[People with disabilities] do not have the money to buy and maintain the hygiene which is currently the most important thing to rescue themselves from the COVID-19." (woman, physical impairment, IWCOVBGA7)

The financial pressures caused the interviewees and their families significant stress as they struggled to meet their and their families' needs (see emotional effect section below).

"My family faced a lot of financial crisis. My mental condition is not good and I'm always stressed." (man, physical impairment, IWCOVBGA4)

This applied not only to our interviewees with disabilities but also to the people with disabilities they knew in their communities.

"My neighbours, friends, and relatives' condition is not good. They are also facing some sort of financial crisis and going through a mental trauma." (woman, visual, IWCOVBDA10)

In order to survive during the worst of the pandemic lockdown, many interviewees and their families relied on savings, taking loans, selling assets such as land, and financial support from other family members or friends. 
"To run my family, we took several loans to buy grocery items. We tried to pay the loan. But it is really burdensome for us to pay the loan." (woman, physical impairment, IWCOVBGA7)

"If [my elder sister] did not help us in our bad time, maybe we would die in the meantime." (woman, albinism, IWCOVBDB8)

However, not everyone had access to these financial coping mechanisms and family and friends were often going through the same difficulties and struggled to help each other.

"The savings in our hand are already used during the COVID-19 shutdown." (woman, albinism, IWCOVBGA8)

"My family financial condition is very poor now. We do not have any relative who could help us in this situation." (woman, albinism, IWCOVBGA8)

Participants also noting the struggle to find jobs, both during and before the pandemic despite qualifications.

"I have completed my graduation already 2 years ago. But I did not manage [to find] a job... My family is currently pressurizing me to getting any job. My family will not be able to bear my cost anymore. It actually always kills me inside." (man, physical impairment, IWCOVBDA4)

"Despite of my qualification, I did not get any job till now. Everyone mocks me to my face... I determined...that I will try my level best ...- if I am unable to get any job I will not keep myself alive." (man, multiple impairments, IWCOVBGA5)

Even though the inability to find work can be a result of disability discrimination, its affects people with disabilities self-esteem, and the participants expressed guilt and discomfort for becoming dependant or for not being able to provide for their dependants.

"When I seek money from my family members they give it to me but treat me differently which is really embarrassing for me." (man, physical impairment, IWCOVBDB4)

"It is really shameful for me to ask money from my family members. My family members did not say anything but their facial expression says a lot." (man, multiple impairments, IWCOVBGB5)

Very few of our interviewees managed to survive the economic shock as a result of more formally provided relief from government, NGOs, OPDs, whereas most received no such assistance as discussed next.

\section{Governance and services}

None of the Bangladesh IW interviewees mentioned receiving social protection income, and it was noted that few people with disabilities generally were provided with government support. There were also complaints that usual assistance was irregular or being provided late. 


\begin{abstract}
"Many people with disabilities do not have a disability allowance card. So they did not receive any support from the government. People with disabilities did not get any new support from the government." (woman, albinism, IWCOVBGB8)

"The government has provided some of the disability allowance but due to COVID-19 the distribution of the allowance has slowed down." (man, multiple impairments, IWCOVBGA2)
\end{abstract}

It was clear that many people with disabilities, including our interviewees, had slipped through the net and did not receive any assistance from formal sources, with serious consequences for their pandemic experiences.

"Many people with disabilities did not receive any relief. They are starving because of the lack of food." (woman, physical impairment, IWCOVBGA3)

Only two interviewees in this study mentioned aid from their OPD. They also noted that while it was helpful it was not enough to meet their family's needs during the pandemic crisis.

"I have received some relief with the help my DPOs but the amount is very much less than the need." (man, multiple impairments, IWCOVBGA2)

Education services were closed due to the lockdown, and there were problems accessing other services such as medical care, with one women attributing the loss of a child to this:

\footnotetext{
"During COVID-19...I gave birth my daughter in February but unfortunately, I lost my child. She died due to medical issues... After my delivery, my health condition was very bad. Due to COVID-19, I could not find any doctor either public or private...no doctors attended their clinics during the pandemic". (woman, physical impairment, IWCOVBDA7)
}

\title{
Participant's geographical location
}

The lockdown restrictions were experienced as being worse in urban than rural areas. People living rurally, could get outside more easily and had space around them; whereas in the urban areas living spaces were more crowded, making it impossible to maintain social distance, service were inaccessible and there were restrictions on public transport. In rural locations people had some scope to do activities to generate income, but in the urban settings there was a tricky balance of risk (starve or go out to earn). On the other hand, in rural areas people with disabilities have less access to hygiene kits, information and services, and less awareness about the impact of COVID-19.

\section{Identity-related impacts}

\section{Gender related impacts}

Before discussing gendered contrasts, it is notable that some respondents thought that the pandemic had affected men and women similarly, with all experiencing shock, fear, loss and worry about survival (see emotional effect section below). 
However, many participants in Bangladesh thought the pandemic circumstances and loss of income caused family tensions and had changed relations in the home between men and women. (Interestingly, perspectives about whether men or women had been more affected were not linked neatly to respondents' genders).

"There is always an unhappy environment in their home." (woman, albinism, IWCOVBGA8)

Traditional gender norms in Bangladesh position men as the predominant breadwinners, which brings a large financial responsibility. When work stopped and they were no longer able to provide for their families, this was perceived to bring a sense of failure, alongside worry about their families' welfare. It was also reported that men found it difficult being at home all day, as they were not used to domestic life, and unable or unwilling to adjust.
"Men's condition was not good. Men stayed at home unoccupied... This situation increased their depression and frustration. It was really tough for the men to not go out." (woman, intellectual, IWCOVBDA9)
"Many of the men stay at home and use social media - their heads always in anger. Many men could not ... have their usual outing with friends. Due to the lack of job, men suffer from mental trauma." (woman, physical impairment, IWCOVBGA3)

On the other hand, women generally have to manage both the family and their jobs simultaneously which creates ongoing pressures. During the lock down they couldn't do their jobs or shop.
"Many women usually do domestic chores for their living, but couldn't due to COVID-19. Besides that, women couldn't go out for necessities at the market or bazar." (woman, physical impairment, IWCOVBGA3)
"Many of the women who worked in garments has lost their job... Many as well as a job. It is really troublesome for most of the women." (woman, physical, IWCOVBDA7). women working from home also have pressure due to managing the family

Some interviewees also noted a changed behaviour towards and by women when they went out:
"Neighbours teased us about using the gloves and mask during outings, but ...never said anything to men. I think there is a different attitude showed by people in the surroundings, who assume men go out for work purposes." (woman, physical impairment, IWCOVBGA3) COVID-19 women are using scarf/Hizab and mask for their precautions." (man, multiple impairments, IWCOVBDA2)
"I think before the COVID-19, some women went outside openly but due to

Stresses manifested for both genders, but within households with less space and financial worries the tensions were amplified, seriously affecting gender relations, and upsetting the 
usual dynamics between spouses, parents and children and other relationships. Many participants spoke of increased conflict at home, felt to be due to men and women being forced together for extended periods (because of curfew, working from home guidelines or partners not having work), and the financial pressures.

"Due to the lack of employment, there is always a quarrel hanging on the family." (man, visual impairment, IWCOVBGA6)

"They may quarrel with their family members without reason because of mental pressure." (man, visual impairment, IWCOVBGA6)

Thus, there were more arguments, and although no interviewees reported direct experience of physical violence, their perception was that it was happening in other households.

"Because of the financial burden, they are suffering from mental illness... and tried to do violent activities at home." (man, visual impairment, IWCOVBGA6)

\section{"Some women experienced domestic violence due to the male family member being at home... Some women face physical torture without any reason." (woman, physical impairment, IWCOVBGA3}

An increase in gender-based violence outside the home was also reported, such as attacks or rape of girls and women. Respondents attributed this to increased stress within homes, and disruption of the norms of social behaviour. Our interviewers also observed that these tensions decreased as men earned income again after the restrictions eased and they could work again. Although the mental impact of the crisis is likely to stay with people for a while.

\section{"The rate of the family violence and gender-based violence has decreased as the male member started their job, business. There is at least little bit earning to run their family." (man, visual impairment, IWCOVBGB6)}

\section{Impairment related}

In addition to the existing challenges faced by people with disabilities and the inaccessibility of social assistance provision, the pandemic has been particularly challenging for people with visual impairments, due to their reliance on touch.

During the pandemic, touch increases the risk of infection and is discouraged to reduce the spread of the virus. Whereas before the pandemic they may have been given assistance by others this was much harder now as people are worried about coming into contact with strangers whose infection status is unknown. Some people in their communities believed that people with disabilities were more likely to be carrying the virus either due to their impairment, or due to inadequate hygiene practices. This resulted in some people with disabilities being ostracised by members of the community. In addition, people with disabilities reported feeling anxious that if members of society contracted the virus that they would be unfairly blamed for it. This resulted in one participant with an intellectual impairment feeling threatened within the home as his parents acted to protect him. 
"My mother scolded me and warned me that if I stepped out from home, I will not be able to enter my house anymore... My father strictly forbade me to step out from house and threatened me that if I step out from home, no one will be responsible for my situation..." (man, intellectual, IWCOVBDA1)

\section{Disability-related stigma}

In Bangladesh, negative stereotyping towards people with disabilities was a strongly expressed theme. Overt expression of discriminatory attitudes was reported by several participants as something they experienced normally that continued during the pandemic.

"I did not face any different treatment from my family or others. But as always I [was] neglected and faced challenges due to my dwarfism." (man, physical impairment, IWCOVBGA4)

Mocking and taunting was a particular problem for those with 'visible' impairments such as physical impairments, such as their facial appearance, dwarfism and albinism.
"Everyone mock about my face. Due to my appearance I have never qualified in a job... Due to the facial disability... Disability is a curse, nobody like the PWDs...the people in our society never thought that we are also human... They thought that we are garbage." (man, multiple impairments, IWCOVBGA5)
"Most of the people are frightened to look at my face. Many of them ignore me due to my appearance. I did not like to attend any social invitation because every [stares] ... Sometime neighbours questioned my parent why they did not take me to the doctor." (woman, albinism, IWCOVBDA8)

Teasing, bullying and street harassment was common, and most participants thought it was no different in the pandemic, but some reported exacerbated stigma outside the home towards themselves or others with disabilities.
"My family and friends always treated me well. Due to COVID-19, from my family I have never been treated differently. But in the outside, some people treated me differently. Sometimes, many people teased me due my physical disability. After the incidents, I console myself that I have to be strong." (woman, physical impairment, IWCOVBGA3)
"I was treated differently since the pandemic started. Like, many of my neighbours did not talk with me properly." (man, multiple impairments, IWCOVBDA2)
"I felt that many other person with disabilities has faced different treatment due to COVID-19... For example, my sister's daughter have the disability problem. Her classmate, neighbours did not behave well with her." (woman, physical, IWCOVBDA7)

The negative stereotyping also resulted in challenges around mobility. In Bangladesh, rickshaw drivers would avoid picking up people with disabilities. 


\section{"As many people think that PWDs carry the corona virus. Still the attitude did not change. Many of the auto-rickshaw and other vehicle driver did not want to take me as I am a disabled person." (woman, physical impairment, IWCOVBGA3)}

The second interviews revealed that negative stereotyping, mocking and bullying has continued even as restrictions have been lifted and some aspects of life were returning to how they were before. Negative attitudes towards people with disabilities is deeply ingrained within Bangladesh, but the study suggests that the pandemic may have exacerbated the stigma experienced by some people with disabilities. Stigma is experienced as very hurtful, but during the pandemic this was compounded by a range of other emotional impacts, as is explored next.

\section{Emotions/feelings (internal or subjective) effects}

As is illustrated in discussing the more concrete themes so far, like many other people worldwide, the participants with disabilities in this study went through and may be continuing to experience difficult emotional journeys due to the COVID-19 pandemic. This was in response to the abrupt suspension of the 'old normal' life, the dramatic changes, and the need to adapt many aspects of normal routines to the new situation.

Initially many participants experienced intense shock and fear, as their usual life was completely disrupted.

\section{"I heard about COVID-19 from television. I felt that it will create inhalation and I felt frightened." (woman, intellectual, IWCOVBDA9)}

"The fear of corona always attacks me like I will be affected with corona any time. It kills me all the time inside... Someone might die for no reason.'. (man, multiple impairments, IWCOVBDA2)

The more existential panic soon diminished for some it replaced by the understandable fear of contagion.

"After heard about the Covid, I felt frightened about it like I will die... After some days, the fear faded away." (man, intellectual, IWCOVBDA1)

"The interaction between neighbours, friends and relatives also decreased. I felt that everyone got frightened due to the COVID-19." (woman, albinism, IWCOVBDA8)

However, many people with disabilities felt people were unfairly afraid of them, which was upsetting.

Loss was a predominant theme. This encompassed the loss of the stability from the interruption of usual lives, resulted in disorientation and uncertainty about what would happen to participants and their families. 
"We could not pass a single good day. My college has closed down. I don't know when the college will open again. My sister's school has also closed." (woman, visual, IWCOVBDA10)

"I am totally stuck in my house. My study is totally stopped. I can't apply for any jobs - there are no new jobs during COVID-19. Everything is totally uncertain right now..." (woman, albinism, IWCOVBDA8).

There was sadness at the loss of opportunities and a sense of disappointment at buried dreams.
"Due to the long shut down, I lost my academic year... If I could have completed my degree...I would have entered employment earlier...to reduce the burden on my family. It actually depressed me a lot." (woman, physical impairment, IWCOVBGA3)

Those whose education was halted also had concerns about their future ability to find work, as in Bangladesh some government job applications are age limited.

"Due to my education stopping, I am concerned about the age limit... I will not be able to join the government as per my interest." (man, multiple impairments, IWCOVBGA2)

Whilst most interviewees understood the need to stay at home to protect themselves, the loss of freedom during lockdown made people unhappy, with some finding it suffocating or feeling imprisoned.

\footnotetext{
"I had to stay at home all the time. I felt bored in that time." (woman, albinism, IWCOVBDA8)

"Many persons with disabilities were not able to step out from home. The children with disabilities did not go their school.... They are prisoned at home... They are facing the problem of suffocation." (woman, visual, IWCOVBDA10)
}

"They are suffering a lot due to stuck in the house. They could not step out from and may be felt suffocated due to the long time stay at home." (man, visual impairment, IWCOVBDA6)

These feelings may have been felt acutely by the participants due to their youth, although further research would need to confirm this. However, this study did suggest that in Bangladesh the lockdown was experienced especially keenly by participants with intellectual impairments, as well as thought to be worse for children with disabilities.

"The condition of the other friends (with Downs syndrome) are same. They are stuck at their house... I feel suffocated. I cannot take breath due to the suffocation and stuck at home." (woman, intellectual, IWCOVBDA9)

"Especially care children with Downs syndrome, intellectual [impairments], or cerebral palsy felt suffocated due to COVID-19. They could not step out from home and they always remain depressed." (woman, albinism, IWCOVBDA8) 
Lockdown and other restrictions were also experienced as making life lonely and isolated.

"The communication and interaction between friends and relatives has totally stopped. There is always a tension in everyone's mind." (woman, physical, IWCOVBDA3)

"There is no communication between the neighbours. Everyone maintains social distance due to the COVID-19." (woman, intellectual, IWCOVBDA9)

Not being able to afford communication costs contributed to interviewees feelings of isolation as it made it hard for them to stay in touch with their friends and OPD members:

"I have to stay at home all the time and I did not able to communicate through phone with my friends as I did not have the ability to buy the talk time." (woman, visual impairment, IWCOVBDB10)

"I could not communicate with the other DPO members through phone due to lack of money." (woman, physical impairment, IWCOVBGA3)

People's usual support structures were also affected which made it harder for them to cope.

"The activities of my DPO have totally stopped... If I could visit my DPO regularly, maybe I could learn some new things which may help in many other ways... By communicating with other members, we could share our problems and it would help lessen the pressure..." (woman, physical IWCOVBDA3)

As previously mentioned, the loss of freedom during lockdown exacerbated tensions between spouses and other family members.

The pandemic lockdowns and other restrictions clearly created much worry and distress for people with disabilities. Inevitably, due to the economic problems they faced, the initial acute fear of the virus was taken over by fear of hunger. People felt hopeless, helpless and very depressed in the worst times of the crisis.

"I almost lost all my hope due to my mental health, physical health and also for the financial crisis." (woman, physical impairment, IWCOVBDA7)

"People with disabilities could not work... Their economic condition is worse right now. It gives me a lot of pain when I think about it." (man, multiple impairments, IWCOVBGA2)

"It gives a lot of mental pressure. Sometimes I felt that the inner pain has traumatized me inside." (man, visual impairment, IWCOVBGA6)

Some people found comfort in religion and trusting to Allah to get them through the situation.

"I always pray to Allah to save us from the COVID-19." (woman, visual impairment, IWCOVBDA10)

"Maintain the hygiene protection, pray to the Almighty for his blessings." (man, multiple impairments, IWCOVBGA2) 
Overall, many strong feelings were generated by the pandemic, and it is important to understand the emotional impacts, as subjective feelings such as frustration or lack of hope are a barrier to people's agency, which is needed in the COVID-19 circumstances to adapt to the 'new normal'.

\section{Changes over time - concrete circumstances and emotional responses}

The second interviews indicated that life for people in their communities had generally improved as restrictions eased, and some people were able to start or find work again. This was true for some interviewees themselves or their families, and for these respondents this covered immediate needs and eased the mental stress and worries. However, many participants and their family members were still unemployed, and the conditions of those who found work again were often worse than before, with lower wages and fewer hours. This meant many people were still struggling to thrive or even survive, and governance financial support still did not seem forthcoming.

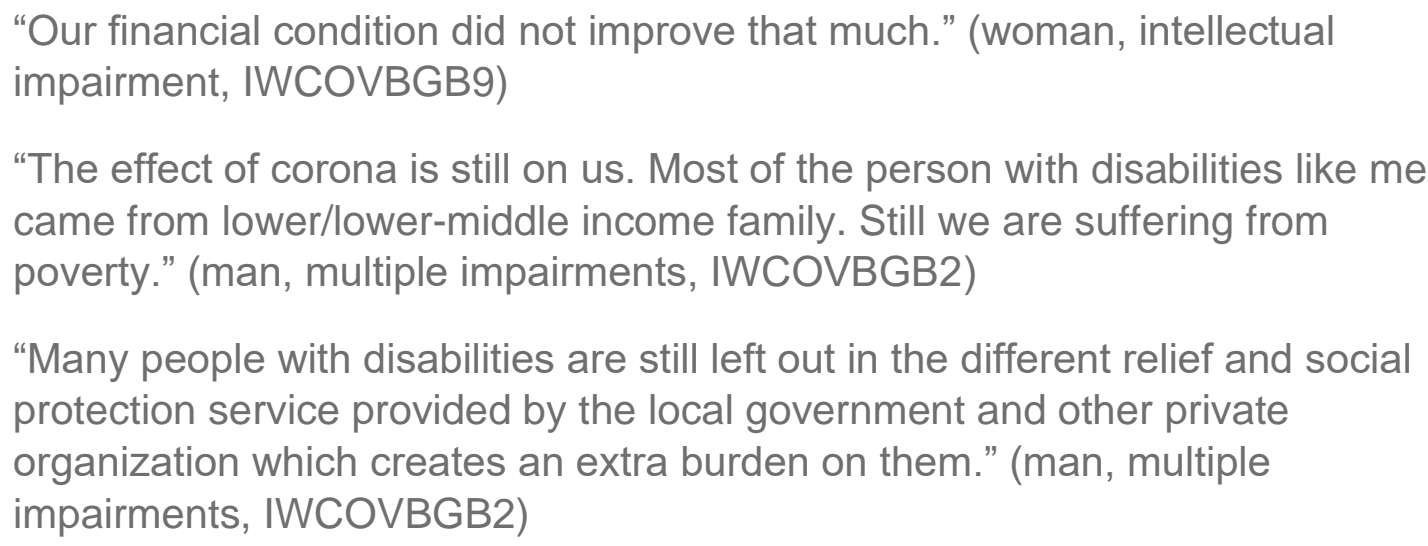

Many of the interviewees with disabilities were unable to find employment despite their best efforts, including as a result of disability discrimination. The interviewers also observed that other people with disabilities in their communities were generally still struggling to find work due to lack of opportunities and they had used up their businesses capital to survive.

\footnotetext{
"My family struggle every single day to run our family. These situations always kill me inside that I could not support my family despite my graduation." (man, physical impairment, IWCOVBGB4)
}

"I tried to overcome the frustration by joining different training program which was arranged by different partners under the IW programme." (man, multiple impairments, IWCOVBGB5)

\footnotetext{
"Many of the person with disabilities are still struggling to start their job or business...they could not able to start due to lack of opportunities... Many of them used a principle amount from their business in their daily livelihood in the last few months." (man, visual impairment, IWCOVBGB6)
} 
As education moved online, the participants who were still studying struggled as they could not afford the necessary technology or data to access their classes.

"My online classes started but I did not attend many classes due to not having internet access. I did not have the money to buy the internet to join the classes." (woman, visual impairment, IWCOVBGB10)

"My college is still closed but some classes are ongoing online. But due to the high data cost, I did not able to attend... It hampers my education." (woman, physical impairment, IWCOVBGB3)

The rise in the price of transport and food was another burden people faced as restrictions eased. For example, one participant's father had got their job back but the family income:

"... is not sufficient as most of the daily commodities price has increased." (man, intellectual impairment, IWCOVBGB1)

Buying medicine also continued to be a problem:
"As I have thyroid and other disability problems, I need regular medicine but I did not have the money... The problem is worsened due to my parent in law's medical cost... I was not able to manage our daily living cost, how could we manage the extra medical treatment cost. Due to this, I and my husband have remained tense." (woman, physical impairment, IWCOVBGB3)

Some interviewees and their families moved from urban to rural areas to cope. Although life was still difficult as there was little work there.
"Due to COVID-19, we have shifted from urban area to rural area as my father did not have the job in that time." (woman, visual impairment, IWCVOBGB10)
"Now we are living on a relative's land where we do not have to pay rent but we have to pay the utility bill which is a burden for us as we still do not have any earning source. Right now, we could not meet our family needs... We have to pass our daily time sometimes without food. There are no opportunities for work as it is the rural area." (woman, visual impairment, IWCOVBGB10

The repayment of loans taken to survive over lockdown was also a major concern and burden for people with disabilities and their families.

"Because of the financial crisis, we had to take loan from others - a very big burden on us." (man, visual impairment, IWCOVBGB6)

"We have to repay our loans which also worsens our situation." (woman, intellectual impairment, IWCOVBDB9).

One family had sold land as a result further decreasing capital: 


\begin{abstract}
"We had to sell many of our village home land to survive as we are four sister in our house... Some of my relatives support us in the last few months as they are observing our hard time passing. In addition to this, we have to repay our loans which also worsen our situation." (woman, intellectual impairment, IWCOVBGB9)
\end{abstract}

The initial fear of COVID-19 may have subsided, but been subsumed by the fear of hunger, with some serious worries about how people would survive a possible second lockdown due to their family's financial situation.

\begin{abstract}
"The second wave of the corona has started in Bangladesh. The total condition is still uncertain for me and my family. I am totally tense about what will happen if the government again announced the lockdown." (man, visual impairment, IWCOVBGB6)
\end{abstract}

\title{
Data validation
}

A joint online data validation event for Bangladesh and Nigeria was held in May 2021, to check the findings resonated with participants' experiences, and to give them the opportunity to share and learn from each other. All interviewees were invited and compensated for data usage to enable participation. Some representatives from NGOs and OPDs involved in the Inclusion Works programme also joined. While not everyone was able to attend and some people had internet issues which meant they were unable to be online throughout, 6 interviewees from Bangladesh and 10 from Nigeria participated. The main findings from the two countries were presented and then participants discussed them in small country groups to consider whether the key themes reflected what had happened to them and if they had anything else to add.

The participants validated the findings through agreeing that the lives of people with disabilities had been negatively affected during the pandemic by insecurity, a lack of social protection, limited health services and hunger. COVID-19 and the national responses had resulted in disruption to their lives, trauma, fear, and stress.

Participants from Bangladesh wanted to emphasise that the impact that COVID-19 had on jobs was particularly devastating for people with disabilities. Opportunities for jobs were already limited and COVID-19 made the situation worse. Income from migrant workers dried up. The financial security of entire families was affected. Job opportunities for people with disabilities became even more limited and some people with disabilities were forced to take work which was not safe and/or did not offer fair pay. Other people had to take loans, some of which had unfair rates or were unregulated.

Access to health care also became difficult during the crisis, with many people with disabilities struggling to access the services they needed. Access to education was also disrupted, and people with disabilities were disproportionately affected. This disruption had a significant impact on the mental health of people with disabilities in Bangladesh. 


\section{Achievements and limitations of the study}

The use of narrative interviewing as a specific type of very flexible qualitative interview allowed for a focus on interviewees' own concerns and helped generate insights on situational, emotional, relational and dynamic aspects that can be missed by researcherdirected interviews. However, this approach was harder for inexperienced researchers than more conventional interviews, which resulted in more direct questioning prompts than intended, especially to some participants with intellectual impairments when the researchers struggled to get communication flowing. Despite this, the study generated rich and deep evidence on the interviewees' feelings, and some insight into the nuances of contextual impacts by gender, impairment and other intersecting influences such as relative poverty or access to assistance. Some participants noted how much they appreciated the process and the opportunity to share and analyse their experiences.

Researchers found interviewees communicated more openly in the second round due to the developing relations between them, as we had hoped. We thought this was also due to both the researchers and participants developing confidence in the research approach and what was expected.

Efforts were made to work with OPDs to recruit a diverse group of people with disabilities but unfortunately it was not possible to recruit participants from all impairment groups. However, there was some success in recruiting participants from groups who are often particularly marginalised or left-out of research such as people with intellectual disabilities or multiple impairments, whose perspectives are not often heard. As the participants were recruited from the Inclusion Works programme, and therefore identified as 'jobseekers', their experiences and perspectives do not represent the whole range of types of people with disabilities in Bangladesh. We were not setting out to be representative. For example, the participants educational level is higher, especially given the high number of college/university students in this cohort, and they have likely had more opportunities than a broader sample from the wider disability community in Bangladesh would have had.

Despite the use of two interviews giving us the opportunity to understand how experiences of the pandemic changed over time, it still is ongoing, so what is presented cannot be the full story of our interviewees' experiences of COVID-19.

Whilst virtual interviews are COVID-19 safe and acceptable approach (see methodology section), interviewing people remotely using online platforms or phones inevitably constrained interactions and was unsatisfactory for some participants and for the interviewers. It also limits participation to those with access to the relevant technology. Although we made great efforts to ensure that the process was inclusive and accessible, including paying for the internet data used so that people could afford to take part, we recognise that it was less inclusive and accessible than a face-to-face process would be. 


\section{Participants' recommendations}

Existing pandemic responses have been criticised for not directly including the perspectives of people with disabilities in their design and implementation, therefore interviewees were asked for their recommendations for responses to COVID-19 and future pandemics. Below are the main recommendations from the participants in Bangladesh. They suggest more disability inclusive responses to the current situation and future pandemics are needed.

As well as participants making recommendations that were pandemic related and disabilityspecific there were also suggestions that were rather general, arguably relevant to nonpandemic situations, and indeed some were not necessarily disability specific. This should be recognised, and in response we have combined and edited the recommendations to some extent.

\section{For the Inclusion Works programme}

- Raise awareness of disability inclusion at all levels to change discriminatory attitudes and reduce social stigma.

- More employer counselling, sensitization and advocacy is needed to encourage recruitment of job seekers with disabilities, and avoid contract termination due to COVID-19 challenges.

- More financial assistance is needed to rehabilitate job seekers with disabilities after the pandemic.

- Job seekers with disabilities should be inspired to actively do something and encouraged that they can achieve.

- Increased advocacy with local and national-level governments to encourage relief and social protection provision to people with disabilities as a priority.

- Provide soft and hard skill training to job seekers with disabilities that is employer needs bases to qualify them for recruitment process.

\section{For local organisations including OPDs}

- Raise the awareness of people with disabilities, and their families and wider communities about COVID-19 safety measures.

- Provide social opportunities for people with disabilities so address isolation and exclusion.

- Decision-making committees should be formed, led by and including OPD members, to ensure that the challenges and issues faced by people with disabilities are considered and communicated to relevant local government departments.

\section{For national government}

- The government should learn from the different initiatives that other countries have used to minimize pandemic losses. 
- Increase transparency and accountability of the ministries and departments who are responsible for relief distribution and other services provided in the pandemic; and create a monitoring system to reduce corruption and bias of the responsible government stakeholders.

- Government and private stakeholders should increase the financial support and relief level to persons with disabilities including money, food, medicine and hygiene kits.

- Special priority should be given to people with disabilities at the grassroots level in distributing different services including medicine and the corona vaccine.

- Health services should be improved with special attention to the needs of people with disabilities.

- Public-Private Partnerships (PPP) should be created to provide services to marginalized and disadvantaged group.

- A disaggregated database should be prepared to determine the losses due to the coronavirus focusing on the marginalized group.

- Financial assistance, including bank loans without interest and rent subsidies, and a livelihoods rehabilitation programme should be provided to people with disabilities

- More job opportunities should be created so people with disabilities can become more economically independent.

- The government should increase the age limit for entering a government job for those who lost an academic year due to the pandemic.

- The quota system for 1 st and 2 nd class government job should be restored so that disadvantaged people with disabilities have a chance.

- The government should give extra consideration to employment opportunities for job seekers with Down syndrome, intellectual impairments or cerebral palsy.

- A special committee should be formed to improve life conditions for people with disabilities and their safety from any type of assault/molestation or abuse.

- Government should implement existing laws and regulations to ensure people with disabilities basic rights.

\section{International Agencies:}

- Advocate to encourage international and Bangladeshi stakeholders to consider and include people with disabilities in the plans and services in response to COVID-19.

- Provide more funds to support people with disabilities build back their lives.

\section{Recommendations to increase preparedness for future pandemics:}

- The government should identify lessons from the COVID-19 pandemic so that responses to any future pandemic can be more timely and effective.

- The government should develop a contingency plan in preparation for future pandemics that gives more priority to people with disabilities.

- Mass awareness should be generated about the importance of maintaining different hygiene measures.

- People should be encouraged to increase savings in readiness for the challenges of a future pandemic. 


\section{Conclusions}

This research has shown that the COVID-19 pandemic has had devastating and lifechanging detrimental effects on people with disabilities' lives in Bangladesh. It has halted their life plans and its consequences will affect their future after the pandemic ends. The participants who took part in our narrative interviews illustrated that the impact of the COVID19 pandemic has been wide-ranging and extreme in some cases. The data shows themes emerging in the lives of people with disabilities due to COVID-19. These include a negative impact on finances, emotional wellbeing, relationships, access to social protection and opportunities.

Many of the impacts were found to interact with each other (for example, losing employment - leading to financial concerns - leading to deteriorating mental health). Some of the impacts affected the whole community, but for people with disabilities the impacts often seem to be exacerbated or compounded by their pre-existing difficulties resulting from barriers and disability discrimination. This reflects findings elsewhere that the pandemic has deepened pre-existing inequalities.

Starting the pandemic from a position of unemployment or insecure employment in the informal sector meant that the financial impacts hit people with disabilities harder, especially as the informal sector was particularly affected by the lockdown and pandemic restrictions. Food insecurity was a major issue, especially for those in urban areas who were unable to grow their own and many people with disabilities and their families were going hungry. The stress of the financial difficulties was very hard for people and caused tensions in many families. For men, not being able to fulfil their role as breadwinner, seemed to cause much anguish and frustration. Relying on family and friends for support was hard as the pandemic's impacts were wide-ranging in their communities and most people were affected. Using up savings or taking loans in an attempt to survive also makes it harder for people to restart their lives when the pandemic ends.

There were clear frustrations and disappointments from our participants with the lack of social assistance provided by the different government social protection programmes. Despite the financial difficulties they faced, most people with disabilities did not receive any COVID-19 aid and regular support was insufficient. While NGOs and OPDs provided support to some, this was often inadequate due to resourcing issues. This left some people feeling disappointed and disillusioned with the lack of practical help from the wider disability movement.

Despite some improvement as restrictions eased, people with disabilities were still struggling. Where possible they were trying to find new work to provide for themselves, but this was challenging in an environment that continues not to be disability inclusive.

To conclude, our data showed that people with disabilities have been marginalised by the pandemic and government responses to it. Inclusive approaches must be developed to ensure that the rights and fundamental freedoms of all persons with disabilities can be realised without discrimination of any kind on the basis of disability. 


\section{Acknowledgements}

Thanks to all the participants and their supporters in Bangladesh who agreed to share their experiences with us, particularly at this difficult time.

We would also like to acknowledge the contribution of the Inclusion Works partners, including Sightsavers, ADD International and local OPDs in each country who helped us greatly with logistics and recruitment of participants and in some cases participated in reviewing our report. They include:

- The team at Sightsavers Bangladesh, especially Utpal Mallick

- The team at ADD International Bangladesh, especially Shafiqul Islam (Country Director), Subodh K. Das (Head of Program) and Dewan Mahfuz E Maula (Coordinator, Policy Advocacy).

- The team at Access Bangladesh Foundation

The members of the Country Implementation Group for their helpful comments and engagements in what was coming out of the interviews.

Thanks to Atlas Translations Ltd. for their translation of the executive summaries.

Thanks also to Caroline Martin and David Haddock for their organisational support.

\section{References}

Ahmed, N., Christensen, A., Cain, E., \& Garbett, A. (2020). COVID-19 Double Jeopardy for Persons with Disabilities. ADD International.

https://add.org.uk/file/4225/download?token=jFGRN8_7

Alif, A. (2020). Covid-19 pandemic: Testing remains low despite having enough kits in stock. Dhaka Tribune. March $29^{\text {th }}$.

https://www.dhakatribune.com/bangladesh/2020/03/29/covid-19-pandemic-testingremains-low-despite-having-enough-kits-in-stock

Braun, V and V. Clarke (2013) Successful qualitative research: a practical guide for beginners, Sage publications Ltd., London, New Delhi

Christensen, A. (2020 a). Covid-19 - Income loss, risk of violence and the response of persons with disabilities and their representative organisations in Uganda. ADD International. https://add.org.uk/file/4321/download?token=U-U2IhDu

Christensen, A. (2020 b). COVID-19: Violence risk and income loss among persons with disabilities. ADD International. https://add.org.uk/file/4271/download?token=j-v793vJ

COVID-19 DRM Disability Rights Monitor. (2020). Disability rights during the pandemic. COVID-19 Disability Rights Monitor. https://covid-drm.org/en/statements/covid-19disability-rights-monitor-report-highlights-catastrophic-global-failure-to-protect-therights-of-persons-with-disabilities 
Devulapalli \& Dantewadia, (2020). Mint Covid Tracker: India's corona trajectory has tapered this week but still remains steeper than Asian peers. MINT, $11^{\text {th }}$ April.

https://www.livemint.com/news/india/india-s-corona-trajectory-tapered-but-stillremains-steeper-than-asian-peers-11586576536468.html

Goyal, N., Raghavan, S., \& Kothan, K. (2020). Neglected and forgotten: Women with disabilities during the Covid crisis in India. Rising Flame \& Sightsavers.

https://www.sightsaversindia.in/wpcontent/uploads/2020/07/NeglectedAndForgotten_RFandSS.pdf

Hammett, D., Twyman, C., \& Graham, M. (2015. Research and Fieldwork in Development. Routledge, UK.

https://doi.org/10.4324/9780203649107

HI. (2020a). COVID-19 in humanitarian contexts: No excuses to leave persons with disabilities behind! Evidence from HI's operations in humanitarian settings. Humanity \& Inclusion (HI). https://reliefweb.int/sites/reliefweb.int/files/resources/COVID$19 \% 20$ in $\% 20$ humanitarian $\% 20$ contexts $\% 20$ -

\%20no\%20excuses $\% 20$ to\%20leave $\% 20$ persons $\% 20$ with\%20disabilities $\% 20$ behind\%21 \%20Evidence $\% 20$ from $\% 20 \mathrm{HI} \% 27$ s\%200perations $\% 20$ in $\% 20$ humanitarian $\% 20$ settings. pdf

HI. (2020b). Rapid need assessment: An inclusive response to COVID-19 in Nepal.

Humanity \& Inclusion (HI).

https://www.fsnnetwork.org/sites/default/files/HI\%20Rapid\%20Need\%20Assessment\% 20-\%20An\%20Inclusive\%20response\%20to\%20COVID-19\%20in\%20Nepal.pdf

Holt, A. (2010). Using the telephone for narrative interviewing: a research note. Qualitative Research, 10 (1), 113-121. https://doi.org/10.1177/1468794109348686

Hydén, M. (2014). The teller-focused interview: Interviewing as a relational practice.

Qualitative Social Work, 13 (6), 795-812. https://doi.org/10.1177/1473325013506247

i2i. (2020). The impact of Covid-19 on persons with disabilities: Insight and stories from

Bangladesh and Kenya. i2i https://www.leonardcheshire.org/sites/default/files/2020-

07/i2i-covid19-survey-accessible.pdf

IDA. (2020). Toward a Disability-Inclusive COVID19 Response: 10 recommendations from the International Disability Alliance. International Disability Alliance.

https://www.internationaldisabilityalliance.org/sites/default/files/ida_recommendation s_for_disability-inclusive_covid19_response_final.pdf

IDA \& IDDC. (2020). World Health Assembly Statement - Covid19 and Disability. Monday 18 May. IDDC.

https:/www.iddcconsortium.net/wpcontent/uploads/2020/05/iddc_ida_statement_eng_ pdf.pdf

ILO. (2020a). COVID-19 and the World of Work: Ensuring the inclusion of persons with disabilities at all stages of the response. ILO. 
https://www.ilo.org/global/topics/disability-and-work/WCMS_746909/lang-en/index.htm

ILO. (2020b) No one left behind, not now, not ever. Persons with disabilities in the COVID-19 response

https://www.ilo.org/global/topics/disability-and-work/WCMS_741287/lang-en/index.htm

Inclusion International. (2020). People with Intellectual disabilities disproportionately

impacted by COVID-19. https://inclusion-international.org/wpcontent/uploads/2020/12/inclusion-international-covid-impact1.pdf

Jovchelovitch, S., \& Bauer M. (2000). Narrative Interviewing. In Gaskell, G., \& Bauer M. (Eds). Qualitative Researching with Text, Image and Sound - A Practical Handbook for Social Research. Sage Publications, UK

Maswood, M. (2020). Six die with coronavirus symptoms, no new case reported in Bangladesh. New Age. 29th March.

https://www.newagebd.net/article/103364/six-die-with-coronavirus-symptoms-no-newcase-reported-in-bangladesh

Nabi M., S., \& Shovon, F. (2020). 20-fold rise in Covid-19 cases in Bangladesh since April 1. Dhaka Tribune. $14^{\text {th }}$ April.

https:/www.dhakatribune.com/health/coronavirus/2020/04/14/20-fold-rise-of-covid-19cases-in-bangladesh-since-april-1

Nagari, A. (2020). 43\% children with disabilities planning to drop out due to difficulties faced in e-education: Survey. Hindustan Times, $18^{\text {th }}$ July 2020.

https://www.hindustantimes.com/education/43-children-with-disabilities-planning-todrop-out-due-to-difficulties-faced-in-e-education-survey/story-

cMy55e6gQ1XDUqxB9U6M6I.html

Nooruddin, I., \& Shahid, R. (2020). Defusing Bangladesh's COVID-19 time bomb. Atlantic Council, 30 th March. https://www.atlanticcouncil.org/blogs/new-atlanticist/defusingbangladeshs-covid-19-timebomb/

OHCHR. (2020). COVID-19 and the rights of persons with disabilities: guidance. OHCHR. https://www.ohchr.org/Documents/Issues/Disability/COVID19_and_The_Rights_of_Per sons_with_Disabilities.pdf

Paul, R. (2020). Bangladesh confirms its first three cases of coronavirus. Reuters. 8 March https://www.reuters.com/article/us-health-coronavirus-bangladesh-idUSKBN20V0FS

Rohwerder, B., Thompson, S., Shaw, J., Wickenden, M., Kayastha, S., Sigdel, A., Akter, F., \& Bosri, R. (2021). "Because of COVID, everything is mess"- understanding the pandemic experiences of people with disabilities in Bangladesh and Nepal through narrative interviews. Institute of Development Studies.

https://opendocs.ids.ac.uk/opendocs/handle/20.500.12413/16521

Sale, J., Polyakov, M., \& Eaton, J. (2020). COVID-19 and mental health: immediate and long-term impacts. Bond Mental Health and Psychosocial Disability Group Briefing. Bond 
https://www.bond.org.uk/resources/covid-19-and-mental-health-immediate-and-longterm-impacts

Tracey, S. J. (2013). Qualitative Research Methods: Collecting Evidence, Crafting Analysis, and Communicating Impact. Wiley-Blackwell. West Sussex, UK

UN. (2020). Policy Brief: A Disability-Inclusive Response to COVID-19. UN.

https://www.un.org/development/desa/disabilities/wp-

content/uploads/sites/15/2020/05/sg_policy_brief_on_persons_with_disabilities_final. pdf

UNCRPD. (2007). Convention on the Rights of Persons with Disabilities. New York: UN.

Accessed -

https://www.un.org/development/desa/disabilities/convention-on-the-rights-ofpersons-with-disabilities.html

Webster, L. (2020). Coronavirus: Why disabled people are calling for a Covid-19 inquiry. BBC News, 4 July 2020. https://www.bbc.co.uk/news/uk-53221435

WEI. (2020). Statement on Rights at the Intersection of Gender and Disability During COVID-19. WEI. https://womenenabled.org/blog/statement-on-covid-19-forendorsement/

WHO. (2020a). Disability considerations during the COVID-19 outbreak. WHO.

https://www.who.int/docs/default-source/documents/disability/eng-covid-19-disabilitybriefing-who.pdf?sfvrsn=963e22fe

WHO. (2020b). Disability and COVID-19: Ensuring no one is left behind. WHO Contributors: Alarcos Cieza, Ren Minghui, Bente Mikkelsen, Somnath Chatterji, Chapal Khasnabis, Michelle Funk, Andrea Guzman, Nathalie Drew, Kaloyan Kamenov

WHO. (2021). COVID dashboard - Bangladesh. WHO.

https://covid19.who.int/region/searo/country/bd 


\section{Annex 1 - IW COVID interviews - participants in Bangladesh}

\begin{tabular}{|c|c|c|c|c|c|c|c|c|c|c|c|c|c|c|c|c|}
\hline \multicolumn{2}{|c|}{ Gender } & \multicolumn{7}{|c|}{ Impairment } & \multicolumn{3}{|c|}{ Age } & \multicolumn{5}{|c|}{ Education* } \\
\hline$\frac{0}{\frac{D}{N}}$ & 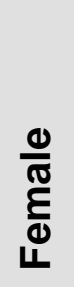 & 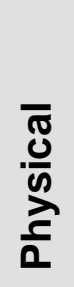 & $\begin{array}{l}\bar{\pi} \\
\stackrel{0}{0} \\
\stackrel{0}{>}\end{array}$ & 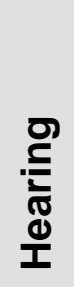 & 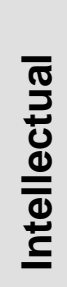 & $\begin{array}{l}\frac{\pi}{0} \\
0 \\
\& \\
0 \\
\frac{0}{0} \\
\frac{0}{0} \\
0 .\end{array}$ & & $\frac{\frac{0}{0}}{\frac{0}{2}}$ & ㄱv & $\begin{array}{l}\text { g } \\
\stackrel{0}{+} \\
\text { 요 }\end{array}$ & ㅇํㅇ & $\begin{array}{l}\text { 0 } \\
\text { 을 }\end{array}$ & 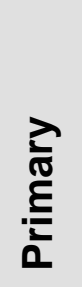 & $\begin{array}{l}\frac{7}{\pi} \\
\frac{\pi}{0} \\
\frac{0}{0} \\
\text { ஸ } \\
\text { ભ }\end{array}$ & 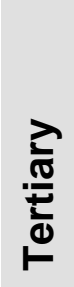 & $\frac{\text { d }}{\frac{c}{0}}$ \\
\hline 5 & 5 & 2 & 2 & 0 & 2 & 0 & 2 & 2 & 9 & 1 & 0 & 0 & 0 & 0 & 8 & 2 \\
\hline
\end{tabular}

\begin{tabular}{|c|c|c|c|c|c|c|c|c|c|c|c|c|c|c|c|}
\hline \multicolumn{4}{|c|}{ Occupation** } & \multicolumn{3}{|c|}{ Marital status } & \multicolumn{3}{|c|}{ Location } & \multicolumn{4}{|c|}{ Dependence ${ }^{* * *}$} & \multicolumn{2}{|c|}{$\begin{array}{c}\text { Times } \\
\text { interviewec }\end{array}$} \\
\hline 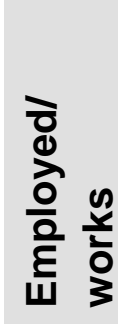 & 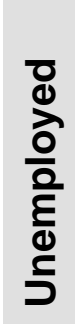 & $\begin{array}{l}\text { 등 } \\
\frac{0}{0} \\
\frac{2}{\partial}\end{array}$ & 迹 & $\begin{array}{l}\text { 언 } \\
\frac{0}{2} \\
\frac{\pi}{2}\end{array}$ & $\frac{\frac{\Phi}{\sigma}}{\text { ஸे }}$ & 高 & 矛 & $\begin{array}{l}\text { 동 } \\
\text { 운 } \\
\text { 일 }\end{array}$ & हे & 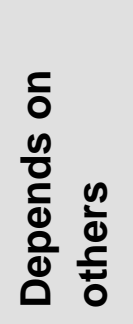 & 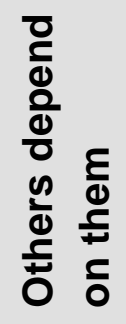 & $\frac{\frac{1}{\Phi}}{\frac{c}{\mathbf{D}}}$ & 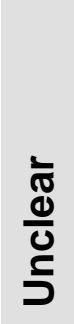 & $\begin{array}{l}\text { ષ્ } \\
\text { ō }\end{array}$ & $\frac{0}{3}$ \\
\hline 1 & 3 & 6 & 0 & 2 & 8 & 0 & 5 & 5 & 0 & 0 & 8 & 2 & 0 & 1 & 9 \\
\hline
\end{tabular}

* Education categories standardised across the four IW countries. College education included under 'tertiary'. Other includes religion-based education and informal education.

** The occupation category of 'other' included volunteers, and those with caring responsibilities who were not actively seeking employment.

${ }^{\star * *}$ The dependence category was open to interpretation by data collectors, but an attempt at standardising the categories across the IW countries has been made. 